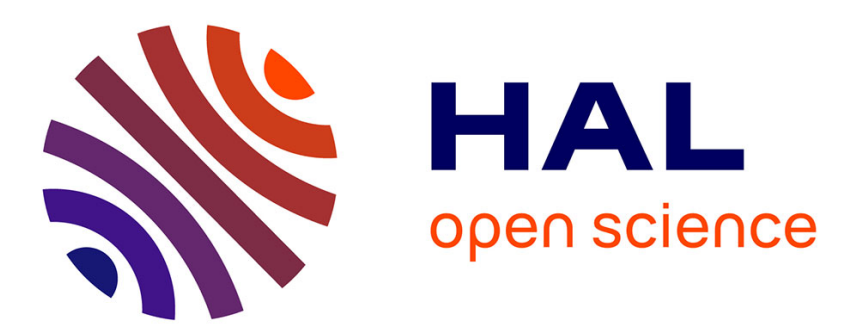

\title{
Coincidence of strain-induced TRIP and propagative PLC bands in Medium Mn steels
}

Michael Callahan, Olivier Hubert, François Hild, Astrid Perlade, Jean-Hubert

Schmitt

\section{- To cite this version:}

Michael Callahan, Olivier Hubert, François Hild, Astrid Perlade, Jean-Hubert Schmitt. Coincidence of strain-induced TRIP and propagative PLC bands in Medium Mn steels. Materials Science and Engineering: A, 2017, 704, pp.391 - 400. 10.1016/j.msea.2017.08.042 . hal-01591751

\section{HAL Id: hal-01591751 \\ https://hal.science/hal-01591751}

Submitted on 21 Sep 2017

HAL is a multi-disciplinary open access archive for the deposit and dissemination of scientific research documents, whether they are published or not. The documents may come from teaching and research institutions in France or abroad, or from public or private research centers.
L'archive ouverte pluridisciplinaire HAL, est destinée au dépôt et à la diffusion de documents scientifiques de niveau recherche, publiés ou non, émanant des établissements d'enseignement et de recherche français ou étrangers, des laboratoires publics ou privés. 


\title{
Coincidence of Strain-Induced TRIP and Propagative PLC Bands in Medium Mn Steels
}

\author{
Michael Callahan ${ }^{\mathrm{a}, *}$, Olivier Hubert ${ }^{\mathrm{b}}$, François Hild ${ }^{\mathrm{b}}$, Astrid Perlade ${ }^{\mathrm{c}}$, \\ Jean-Hubert Schmitt ${ }^{\mathrm{a}}$ \\ ${ }^{a}$ MSSMat, CNRS, CentraleSupélec, Université Paris-Saclay, 91190 Gif-sur-Yvette, \\ France \\ ${ }^{b}$ LMT, ENS Paris Saclay, CNRS UMR 8535, Université Paris-Saclay, 94235 Cachan \\ Cedex, France \\ ${ }^{c}$ ArcelorMital Global RËD - Automotive Products, Maizieres, Voie Romaine, 57583 \\ Maizieres-les-Metz, France
}

\begin{abstract}
An Fe-0.2C-5Mn-2.5Al medium manganese steel is studied for three different intercritical annealing temperatures $T_{I A}$ to observe the effects of variations of retained austenite stability on the mechanical work hardening in uniaxial tension. Digital image correlation (DIC) showed that both Lüders bands and type A Portevin-le-Châtelier (PLC) bands occurred in samples with a lower $T_{I A}$, but both disappeared when $T_{I A}$ was increased. In-situ measurements of sample magnetization with a correction for stress effects on the magnetic properties of the sample allowed for continuous measurement of the retained austenite volume fraction. This method provided a complete characterization of the kinetics of transformation-induced plasticity (TRIP) in the sample over the course of tensile testing. It is shown that the martensite transformation was purely strain-induced and coincided with the passage of both Lüders and PLC bands. An Olson-Cohen model was applied to magnetic data to attempt to explain the differences in TRIP kinetics with varying $T_{I A}$.
\end{abstract}

Keywords: Digital image correlation, Magnetization measurement, Medium Mn steel, Portevin-le-Châtelier bands, TRIP

\footnotetext{
${ }^{*}$ Corresponding author

Email address: michael.callahan@centralesupelec.fr (Michael Callahan)
} 


\section{Introduction}

Increasingly stringent vehicle emissions standards and a high consumer demand for fuel efficient vehicles have led to the development of a third generation of advanced high strength steels (AHSS) with complex multiphase microstructures. One such alloy family is Medium Manganese steels. Medium Mn steels are composed of an ultra-fine-grained (UFG) mixture of ferrite, retained austenite, and in some cases martensite. They can achieve high ductility and high work hardening due to transformation-induced plasticity (TRIP) or twinning-induced plasticity (TWIP) in the austenite phase, which locally harden the steel and delay mechanical instability [1-3]. Previous studies on Medium Mn steels have shown total elongation values of up to $70 \%$ and ultimate tensile strengths (UTS) ranging from 800 to $1500 \mathrm{MPa}$ [4-8]. These steels are intended for high formability in that an initially ductile steel can be hardened significantly during the forming process, thereby achieving mechanical strength criteria without complicating the forming process. Despite this potential, these steels present some challenges for forming operations because the adiabatic heat generated at high strain rates could suppress the TRIP effect, resulting in a loss of hardening capacity.

In order to create an UFG microstructure, the steel is generally homogenized and quenched before cold-rolling to obtain a large fraction of martensite. If the cold-rolled steel is then annealed in the intercritical ferrite + austenite domain, austenite will nucleate at the intersections of martensite laths. The resulting sub-micrometer austenite grain size makes it possible to thermodynamically stabilize the austenite at room temperature with Mn additions of only $5-10 \mathrm{wt} \%$ rather than the $20 \mathrm{wt} \%$ or more needed in TWIP steels $[9,10]$. The retained austenite thus obtained is metastable at room temperature and can undergo TRIP if sufficient mechanical energy is applied. However, the high strain rates used in forming operations can stabilize the retained austenite via adiabatic heating and result in a significant loss in hardening capacity. This illustrates the need to understand how the stability of the austenite is related to the kinetics of the martensite transformation during TRIP. This study will seek to establish a method of characterizing the TRIP kinetics that could be used in future work to optimize compositions and high strain rate performance of TRIP steels.

Proper characterization of the kinetics of the TRIP effect in a particular alloy requires the volume fraction of retained austenite to be measured as a function of strain. This is notoriously difficult to do, however, as dif- 
ferent retained austenite volume fraction measurement techniques can give very different values for similar alloys $[11,12]$. Determination of the austenite content is nonetheless critical in alloy design, as it would provide the metallurgist control over the strain hardening contributions from TRIP and TWIP. Attempts have been made to measure the austenite volume fraction as a function of strain by X-ray diffraction (XRD) [13] and magnetization measurements using a ferritescope [13-15] in order to quantify TRIP kinetics. While in terms of the accuracy of the measurement method, magnetic measurements provide no advantage over other methods [12], the primary interest of the method as employed herein is the extremely high sampling rate compared to, for example, XRD or EBSD. It enables one to measure the retained austenite volume fraction in-situ without having to pause the mechanical test, which would not be possible if one wishes to perform the test at the very high strain rates used during stamping operations for example. Sampling rates this high are not normally obtainable by other in-situ methods, making magnetic saturation measurements the unique choice for such an application if the experimenter wants intermediate points between the unstrained and ruptured states. Moreover, a more complete description of the kinetics of TRIP using several hundreds or thousands of experimental data points would make it possible to refine existing TRIP models used in simulations of mechanical behavior.

Recent studies on Medium Mn steels have noted that strain hardening is sometimes unstable and that long Lüders plateaus are often observed in alloys containing low levels of aluminum $[4,16]$. Additionally, for certain compositions and heat treatments, serrations have been observed in plots of $\frac{d \sigma}{d \varepsilon}$ versus either true stress or true strain. Gibbs et al. [7] suggested that these serrations are due to a variable martensite transformation rate resulting from the competition between the consumption and creation of martensite nucleation sites during plastic deformation. Lee et al. [17] attributed the serrations to dynamic strain aging (DSA) leading to the formation of Portevin Le-Châtelier (PLC) bands. Cai et al. [5] instead proposed that the stability of the austenite phase itself varied from one grain to another and thus could transform to martensite in bursts.

Digital image correlation (DIC) has historically been a common method for characterizing strain localizations such as PLC [18-27]. As such, it is an ideal tool for investigating the jerky flow present in Medium Mn steels [28, 29]. This paper will seek to use a combination of DIC and in-situ measurements of the retained austenite content via the sample's saturated magnetization 
to demonstrate that the observed strain instabilities coincide with the TRIP effect.

\section{Materials and Methods}

\subsection{Fabrication and Microstructural Characterization}

A Medium Mn steel containing $0.2 \mathrm{wt} \% \mathrm{C}, 5 \mathrm{wt} \% \mathrm{Mn}$ and $2.5 \mathrm{wt} \% \mathrm{Al}$ was fabricated in order to study the effects of the heat treatment process on the microstructure and active deformation mechanisms in the steel. Ingots were cast and then hot-rolled at austenitizing temperature to produce a homogeneous austenitic microstructure. They were then quenched and cold-rolled, resulting in a mixture of deformed martensite and bainite. Finally, the steel was annealed in the intercritical ferrite + austenite region at temperatures of $\mathrm{T}_{\mathrm{IA}}=740^{\circ} \mathrm{C}, \mathrm{T}_{\mathrm{IA}}=760^{\circ} \mathrm{C}$, or $\mathrm{T}_{\mathrm{IA}}=780^{\circ} \mathrm{C}$ for 2 minutes. During the intercritical anneal, the deformed microstructure will recrystallize and partially transform to create an UFG mixture of ferrite and austenite in which some retained austenite will remain stable after being air-cooled to $400^{\circ} \mathrm{C}$ and then quenched to room temperature. A representative microstructure of the sample annealed at $740^{\circ} \mathrm{C}$ is provided in Figure 1a for the unstrained state and in Figure $1 \mathrm{~b}$ after straining until rupture. The variations in intercritical annealing temperature result in samples with varying retained austenite stability and initial phase volume fractions. As the retained austenite is metastable, it can transform to $\alpha^{\prime}$ (bct) martensite when mechanically loaded, thereby resulting in high strain hardening ${ }^{1}$.

\footnotetext{
${ }^{1}$ Sometimes $\epsilon$ (hcp) martensite appears as an intermediary phase between $\gamma$ and $\alpha^{\prime}$. In the material studied, no $\epsilon$ martensite was observed in TEM or XRD so it is assumed that the retained austenite transformed directly to $\alpha^{\prime}$ martensite when plastically strained. If $\epsilon$ martensite were present, the magnetic measurements would include it within the ferromagnetic volume fraction (not with the retained austenite).
} 


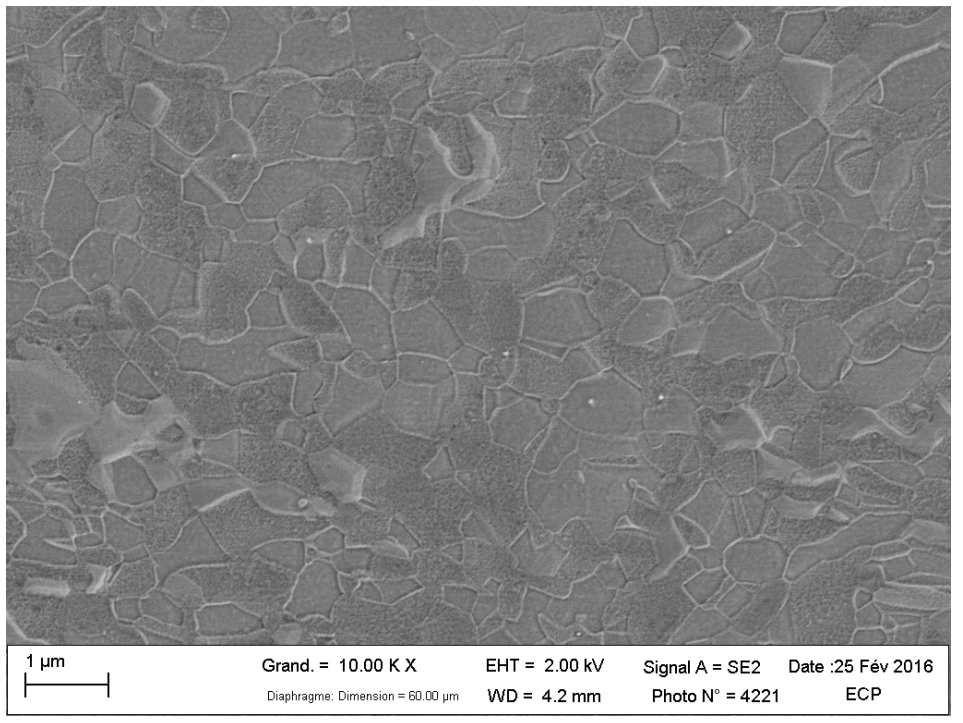

(a)

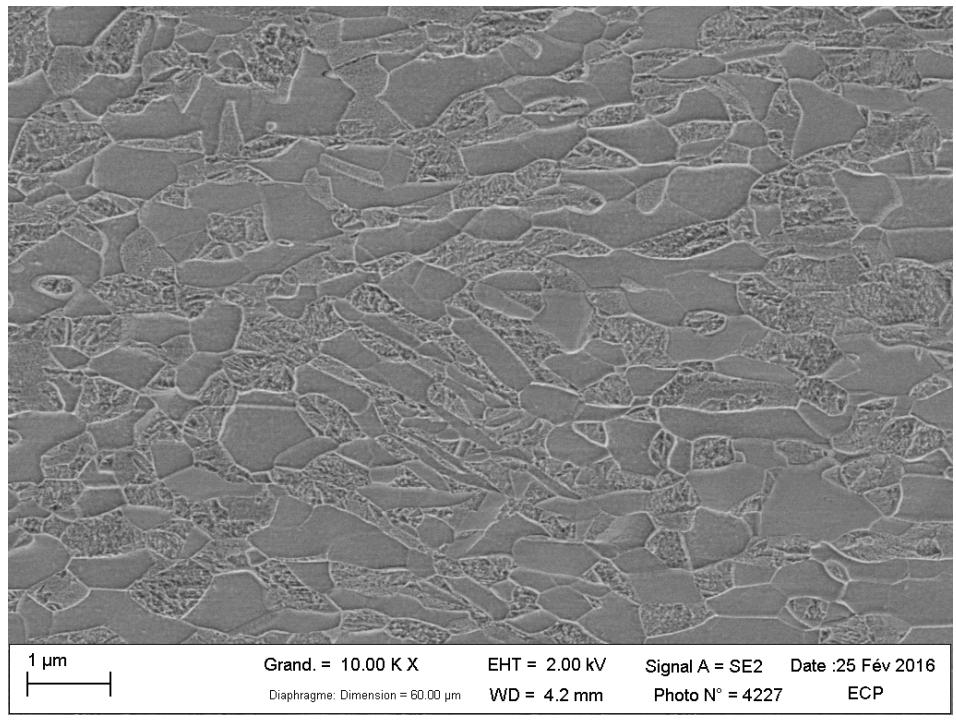

(b)

Figure 1: (a) Microstructure of a sample annealed at $740^{\circ} \mathrm{C}$ in the unstrained state. The microstructure consists of ultra-fine-grained ferrite (the "smooth" phase in the image) and retained austenite (phase with a rougher surface topography) with a roughly isotropic morphology. (b) Microstructure of a sample annealed at $740^{\circ} \mathrm{C}$ after straining to rupture. A significant portion of the retained austenite has transformed to blocks of acicular martensite. The images are taken in the plane of the rolling direction (horizontal) and normal direction. Tension was applied in the rolling direction. 
XRD was used to determine the initial austenite volume fraction for each intercritical annealing temperature. X-rays were produced with a Co- $K_{\alpha}$ source with an energy of $6.915 \mathrm{keV}$. As XRD is a surface measurement, the sample was oscillated in order to increase the volume of measurement (still at the surface) by scanning over a larger area. This provides better statistical sample size in terms of the number of grains analyzed. Spectra were obtained for several orientations of the sample relative to the incident beam. The inclination angle of the sample with respect to the incident beam, $\psi$, was varied from $0^{\circ}$ to $70^{\circ}$ with a step size of $5^{\circ}$. The rotation of the sample around its normal to the sample surface, $\Phi$, varied from $0^{\circ}$ to $355^{\circ}$ with a step of $5^{\circ}$. These spectra were summed together in order to minimize texture effects on the relative peak intensities of the bcc and fcc phases, which can vary significantly depending on the orientation of the sample. The volume fraction of retained austenite was calculated using the method presented in Ref. [30] and the phase volume fractions for each annealing temperature are summarized in Figure 2.

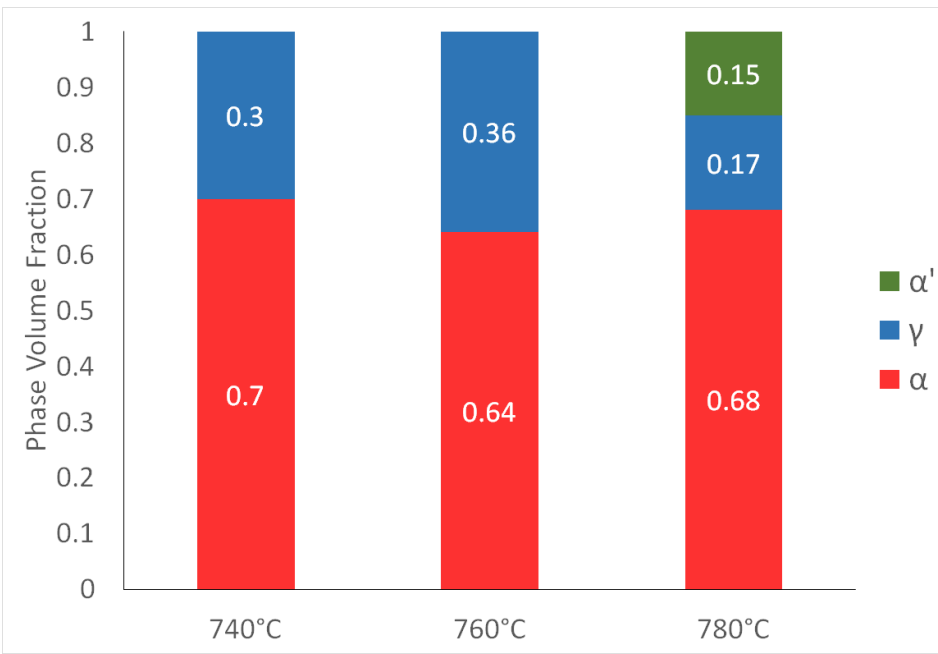

Figure 2: Initial phase volume fractions in the unstrained state after annealing and quenching as measured via XRD ( $\alpha$ and $\gamma)$ and as estimated from SEM images $\left(\alpha^{\prime}\right)$ as a function of the intercritical annealing temperature used.

\subsection{Tensile Testing and DIC}

Tensile dog bone specimens were cut along the rolling direction from $1.25 \mathrm{~mm}$ thick sheet. The specimens had an active gauge length $60 \mathrm{~mm}$ long 
and $10 \mathrm{~mm}$ wide. Unidirectional tensile tests were performed using an Instron extensometer with a $12.5 \mathrm{~mm}$ initial length. The samples were strained until rupture using an Instron 4430 electromechanical tensile testing machine with a strain rate in the sample of $5 \cdot 10^{-4} \mathrm{~s}^{-1}$.

Images for DIC analyses were obtained by painting the polished sample surface with a black and white speckle pattern and taking pictures every $3 \mathrm{~s}$ during testing. A single Canon $60 \mathrm{D}$ camera with a $105 \mathrm{~mm}$ macro objective lens was used to acquire the images with an exposure time of $\frac{1}{320} s^{-1}$, ISO grade of 800 , and an aperture f-stop of $\mathrm{f} / 9$. Two halogen lamps were used to illuminate the specimen surface. The images recorded were $3465 \times 5202$ pixels in definition with 16bit encoding and were cropped to remove excess empty space and converted to .tif format for registration purposes. Correlation of the images was done using 3-node triangular elements and regularization using RT3-DIC [31]. Regularization, as used herein, is a means of reducing uncertainties in the displacement fields measured by DIC. It does this through the use of an elastic constitutive law that aids the convergence of the displacement field to mechanically admissible values. A 10pixel element size and regularization length of 100pixels were used for the calculations. Pixel size varied between 0.025 and $0.05 \mathrm{~mm}$ from one test to another and this scale was determined using the measured width of the sample. The displacement fields in photo $n$ were calculated with respect to the reference image using the results from photo $n-1$ to initialize the calculation. That is, Figures 8 and 9a report the nominal strain fields calculated using the first image as the reference image. Figures 8 and $9 \mathrm{~b}$ show the strain rate fields computed with the (Lagrangian) velocity field.

When Lüders and/or PLC bands were observed, the width and angle of strain bands were characterized using ImageJ [32]. Images of the longitudinal strain field where the bands were clearly defined were selected and 8 line plots of the strain level were analyzed along the tensile direction in each image. The resulting peaks in the line profiles were fitted with Gaussian functions. The full width mid height (FWMH) was used to calculate band width and the peak locations in each of the 8 profiles were used to calculate the band angle. Band width measurements are corrected for the displacement of the band during the $3 \mathrm{~s}$ interval between images. The band velocity is calculated using the shift in position of the strain peaks from one image to another.

\subsection{Magnetic Measurements of Austenite Volume Fraction}

A measurement system was developed based on Ref. [33] in order to per- 
form in-situ measurements of the retained austenite volume fraction during tensile testing. While the setup used is applied to flat tensile samples, it could easily be adapted to other geometries for either uniaxial or biaxial tests [34]. The sizes of the coils can be made larger or smaller as needed (as long as one is careful to note the number of loops in the coil as that will be needed for magnetostatic calculations and to calibrate the applied field using, for example, a Hall effect H-coil). The primary consideration when changing sample geometries is that of the measurement depth, or "skin depth", $e$, which is related to the frequency in the driving coil by

$$
e=\frac{1}{\sqrt[2]{\pi f \sigma \mu}}
$$

where $\sigma$ is the electrical conductivity of the sample and $\mu$ its magnetic permeability. In the current case, this provides an estimation of the skin depth of $8 \mathrm{~mm}$ which is several times greater than the thickness of the sample and ensures that the measure is volumetric. For this particular sample geometry, the frequency could be increased to $200-300 \mathrm{~Hz}$ while still maintaining a volumetric measurement. It should be noted that this would affect the measurement in other ways, however, such as increasing the noise in the signal.

The setup consists of a primary coil placed around a tensile sample that creates a magnetic field in the sample. A schematic of the setup is provided in Figure 3. The magnetic response of the sample is then measured using a pick-up coil wound around the sample surface. The field is enclosed by two high-permeability ferrimagnetic yokes used to contain the magnetic field and concentrate it in the sample. The pick-up coil measures the average magnetic flux in the portion of the sample around which the driving coil is placed. In the current setup, this corresponds to a measurement over $37 \mathrm{~mm}$ of the $60 \mathrm{~mm}$ active tensile length of the sample. The measurement is performed at low frequency (here $2 \mathrm{~Hz}$ ) in order to ensure that no eddy currents are present and that the penetration depth of the measurement is sufficient to include the whole thickness of the sample. It must be noted that because the driving coil encloses the sample, it is not possible to perform DIC and magnetic measurements simultaneously on the same sample with this method. Instead, the plastic strain in tensile experiments is compared with the plastic strain from magnetic measurement experiments and the presence of bands is indicated by plateaus that manifest in the extensometer data. While specific bands cannot be correlated to specific increases in martensite 
fraction, the existence of bands in both tensile and magnetic data over the same ranges would strongly indicate their coincidence.

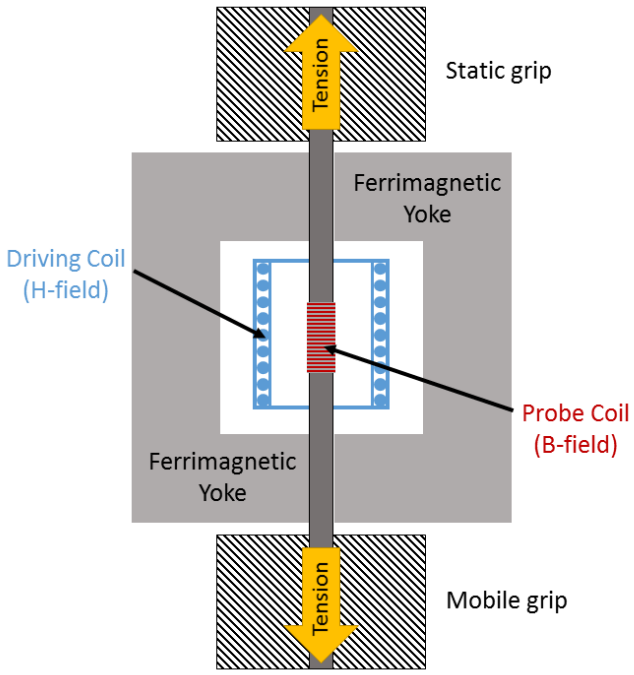

Figure 3: Schematic of the magnetic measurement system. A primary coil (H-field) placed around the tensile sample induced a magnetic $H$ field in the sample, and the current thereby induced in the sample is measured by the probe coil (B-field) and converted to magnetization, $M$. The coils are enclosed in two ferrimagnetic yokes to contain the magnetic fields.

By cycling the current applied to the driving coil at a frequency of $2 \mathrm{~Hz}$, hystereses can be obtained using the readings from the pick-up coil with which the magnetic properties of the sample are analyzed. In this method, as it is typically employed for retained austenite measurements, the saturation value of the magnetization, $M^{s}$, is used to determine the combined volume fraction of the ferromagnetic phases (here ferrite and martensite) using a simple mixture rule

$$
M^{s}=f_{\alpha+\alpha} M_{\alpha+\alpha^{\prime}}^{s}+f_{\gamma} M_{\gamma}^{s}
$$

where ferrite, martensite, and austenite are represented by the subscripts $\alpha, \alpha^{\prime}$, and $\gamma$ respectively. The value of $M_{\gamma}^{s}$ is assumed to be 0 due to the austenite's negligible magnetic response compared to ferrite or martensite. Assuming that $M_{\alpha}^{s}=M_{\alpha^{\prime}}^{s}=M_{\alpha+\alpha^{\prime}}^{s}$, one can express the ferromagnetic volume fraction as 


$$
f_{\alpha+\alpha^{\prime}}=\frac{M^{s}}{M_{\alpha}^{s}}
$$

where $M^{s}$ is the measured saturated magnetization for a given strain level, the subscript $\alpha$ denotes a value measured in a $100 \%$ ferromagnetic sample, and $f_{\alpha+\alpha^{\prime}}$ the combined volume fraction of ferrite and martensite. Figure 4 shows the $M-H$ hystereses thus obtained over $37 \mathrm{~mm}$ of the $60 \mathrm{~mm}$ tensile length for increasing levels of plastic strain for a sample annealed at $780^{\circ} \mathrm{C}$. It can been seen that as the plastic strain increases, the magnetostatic energy in the sample also increases (visible in its integral and in the value of $M^{s}$ ) due to the martensitic transformation.

Equation (3) is frequently used to calculate the retained austenite volume fraction from ferritescope measurements $[14,35,36]$, but it is not suitable for in-situ measurements at stresses exceeding 100-200 MPa due to stress effects on the sample's magnetic properties [37]. The value of $M_{\alpha}^{s}$ usually comes from a reference measurement and is not called into question. $M^{s}$ is more questionable. Figure 5 shows, for example, a clear sensitivity of the magnetization to an applied stress in unloading during which no phase change occurs. This phenomenon, which is known as the Villari effect [38], must be taken into account. Equation (3) assumes that the true saturation is reached during the experiments. This is not achieved in Figure 4. This unsaturated situation leads to some localization effects (i.e. the magnetic field in the ferromagnetic phase is different from the applied field). Two kinds of corrections must consequently be considered as discussed in the following sections. 


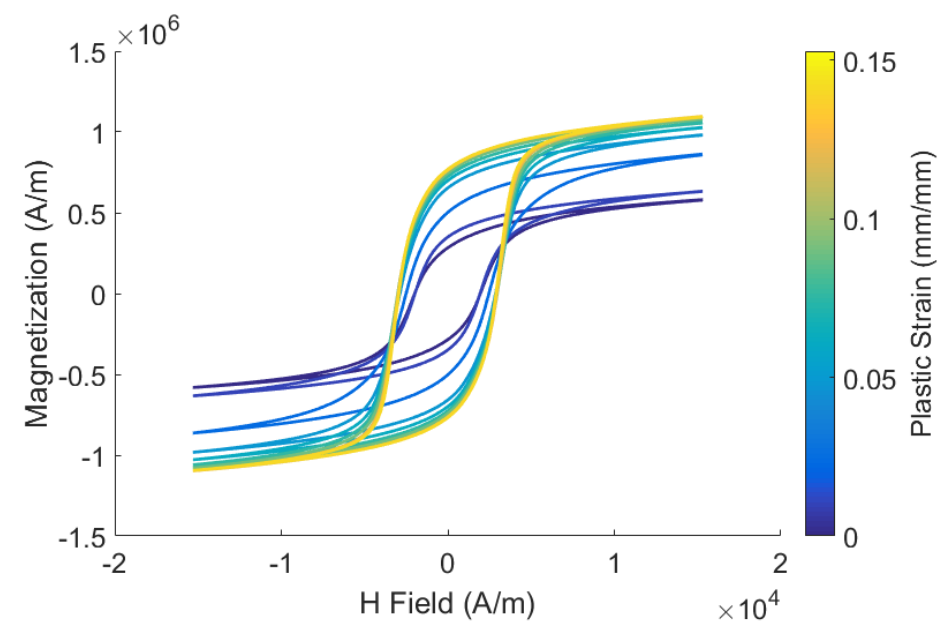

Figure 4: $M-H$ hystereses obtained during unloading of a sample annealed at $780^{\circ} \mathrm{C}$ after several levels of plastic strain (denoted by the color bar) where $M$ is the sample magnetization and $H$ the applied magnetic field. The saturation value of magnetization, $M^{s}$, increases with increasing plastic strain due to the martensite transformation.

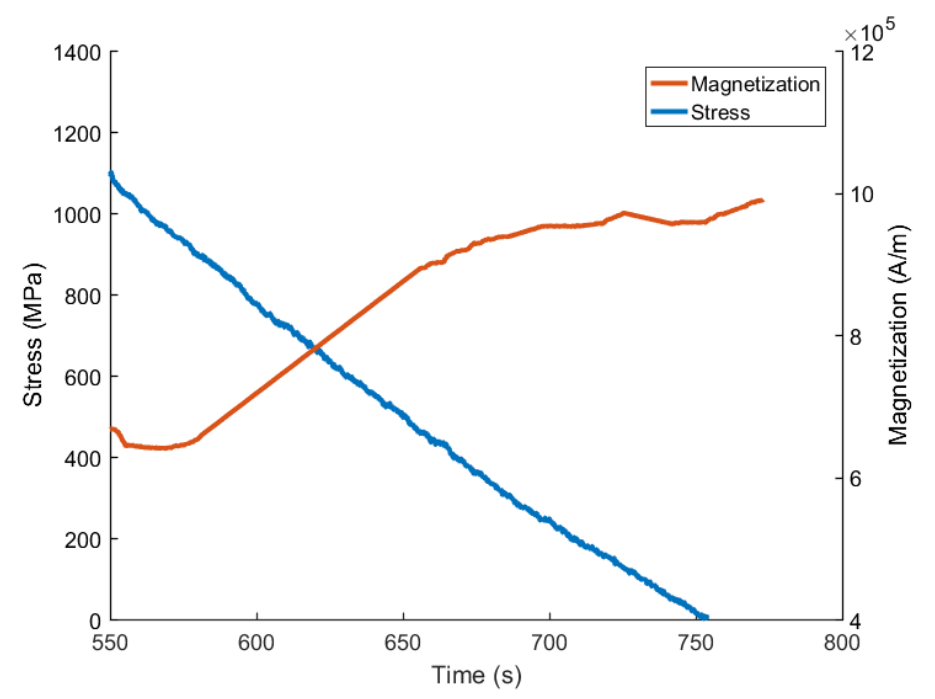

Figure 5: In-situ magnetization measurements of a sample annealed at $760^{\circ} \mathrm{C}$ during unloading after straining until a point just before necking. During unloading, the microstructure does not change (i.e. no TRIP occurs), yet the magnetization increases. This is a result of the Villari effect. The magneto-mechanical coupling demonstrated herein must be corrected if measurements are to be made under an applied stress. 


\subsubsection{Stress Correction}

If measurements of the sample magnetization are to be made in-situ, then the effect of the applied stress on the magnetic properties must be taken into account since it has been shown that even modest loads can have a non-negligible impact on magnetization [39]. A model has been recently developed, which was used to predict the magneto-mechanical coupling in dual phase steels for stresses up to approximately $600 \mathrm{MPa}$ by relating kinematic hardening to magneto-elastic effects occurring within each phase at the grain scale [38]. This approach has been adapted to the current study to correct for the Villari effect.

The multidomain model used here was first introduced in Ref. [40]. It is able to model the magnetization and the magnetostrictive strain of an isotropic polycrystalline ferromagnetic material submitted to a uniaxial applied stress. Two scales are involved: the domain scale (6 domains are considered) and the grain scale taken as an equivalent polycrystalline medium. Macroscopic and crystallographic frames of reference are coincident (i.e. $(\mathbf{x}, \mathbf{y}, \mathbf{z})=([100],[010],[001]))$. In the simplified description we propose here, the rotation of the magnetization is not considered. The stress tensor $\boldsymbol{\sigma}$ and magnetic field $\mathbf{H}$ are assumed to be homogeneous. The free energy of a domain $\phi$ then becomes a sum of the magnetostatic (Zeeman) and magnetoelastic energies:

$$
W_{\phi}=-\mu_{0} \mathbf{H} \cdot \mathbf{M}_{\phi}-\boldsymbol{\sigma}: \boldsymbol{\epsilon}_{\phi}^{\mu}
$$

where $\mu_{0}$ is the magnetic permeability of vacuum, $\mathbf{M}_{\phi}$ is the magnetization vector inside a domain such that $\left|\mathbf{M}_{\phi}\right|=M_{\alpha}^{s}$, and $\boldsymbol{\epsilon}_{\phi}^{\mu}$ the magnetostrictive strain tensor (see details in Ref. [40]). The volume fraction of a domain $f_{\phi}$ is calculated from Boltzmann statistics:

$$
f_{\phi}=\frac{\exp \left(-A_{s} W_{\phi}\right)}{\sum_{\phi} \exp \left(-A_{s} W_{\phi}\right)}
$$

where $A_{s}$ is a fitted parameter proportional to the magnetic initial susceptibility $\chi^{0}$ without an applied stress $\left(A_{s}=\frac{3 \chi^{0}}{\mu_{0} M_{\alpha}^{s^{2}}}\right)$. The magnetization of the single crystal is obtained from an averaging operation

$$
\mathbf{M}=\sum_{\phi} f_{\phi} \mathbf{M}_{\phi}
$$


when the magnetic field and uniaxial stress $\sigma$ are applied along $\mathbf{x}$. After simplifying and applying a second order Taylor expansion of the exponential, one obtains

$$
M=\mathbf{M} \cdot \mathbf{x}=\chi_{0} H\left(1+\frac{6 \chi_{0}}{\mu_{0} M_{\alpha}^{s^{2}}} \lambda \sigma\right)
$$

where $\lambda$ is a global magnetostriction constant. This relationship permits a new definition of the initial susceptibility in which it is linearly related to the magnitude of stress as

$$
\chi_{\sigma}^{0}=\frac{M}{H}=\chi^{0}\left(1+\frac{6 \chi^{0}}{\mu_{0} \cdot M_{\alpha}^{s^{2}}} \lambda \sigma\right)
$$

This equation applies for the initial susceptibility but is assumed to also be applicable for the secant susceptibility at any magnetic field level and particularly at the magnetic field level at which the experimental saturation of magnetization is measured. A linear correction of the stress effect is then obtained

$$
\chi_{\sigma}=\chi(1+\eta \sigma)
$$

where $\eta$ is a constant that can be identified using magnetization measurements obtained during elastic unloading (see Figure 5) without any phase transformation. The constant $\eta$ is typically on the order of $-10^{-5} \mathrm{MPa}^{-1}$. This relationship between susceptibility and stress applies to the magnetization level as well, so a stress-sensitive "saturated" magnetization is defined as

$$
M_{\sigma}^{s}=f_{\alpha+\alpha^{\prime}} \chi_{\sigma} H=f_{\alpha+\alpha^{\prime}} M_{\alpha}^{s}(1+\eta \sigma)=M^{s}(1+\eta \sigma)
$$

Equation (3) is thus modified to become

$$
f_{\alpha+\alpha^{\prime}}=\frac{M_{\sigma}^{s}}{(1+\eta \sigma) M_{\alpha}^{s}}
$$

which provides a correction to the ferromagnetic volume fraction $f_{\alpha+\alpha^{\prime}}$ for a magnetic saturation value $M^{s}$ obtained with applied stress $\sigma$.

The effect of the stress correction is shown in Figure 6 for a sample annealed at $780^{\circ} \mathrm{C}$. This linear correction of the $M^{s}$ value improves the accuracy of retained austenite measurements at high stress where differences of 
up to 0.05 in $f_{\alpha+\alpha^{\prime}}$ were observed between calculations using $M^{s}$ and $M_{\sigma}^{s}$. It also makes it possible to determine whether or not stress-induced martensite transformation occurs.

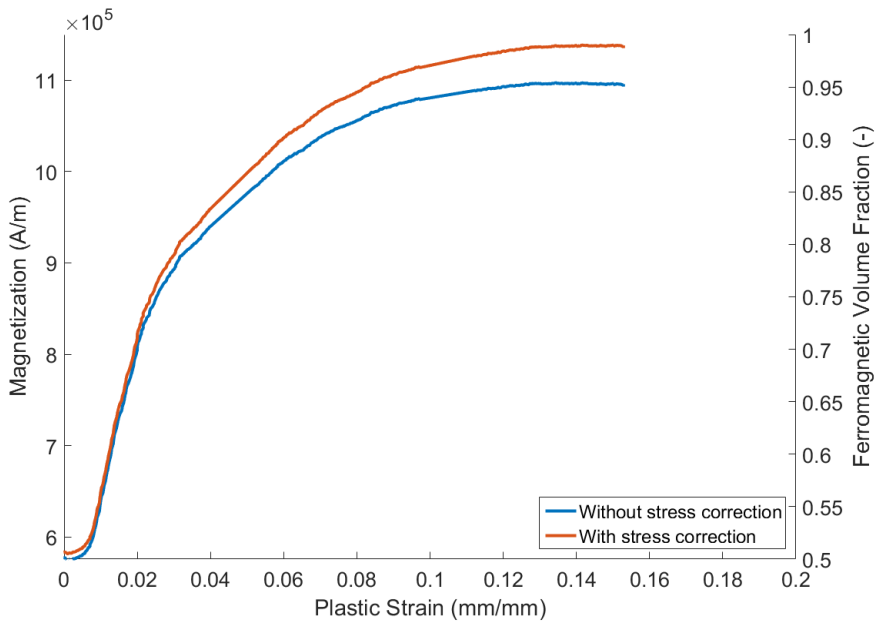

Figure 6: Magnetization and volume fraction of ferromagnetic phases (ferrite + martensite) for $T_{I A}=780^{\circ} \mathrm{C}$ as functions of plastic strain as calculated from in-situ data with and without the stress correction developed in this study. High levels of stress reduce the measured magnetization and result in several percent difference in calculated volume fractions.

\subsubsection{Localization Correction}

The ratio presented in Equation (3) is the most commonly used method of calculating the ferrite + martensite volume fraction using magnetic measurements. However, these equations require the assumption that the field $H$ is uniform throughout the microstructure, which is not true as the negligible magnetic response of the austenite creates a demagnetizing field in the ferromagnetic phase [41], which changes the magnetic properties of the macroscopic sample. An Eshelby model for a spherical inclusion embedded in a homogeneous matrix can be used to localize the field $H$ within each phase and thus correct for the effect of the demagnetizing field on the magnetic susceptibility $\chi$ of the sample $[42,43]$. If the magnetic behavior in a phase $i$ is described by

$$
\mathbf{M}_{i}=\chi_{i} \mathbf{H}_{i}
$$

then the solution to the Eshelby problem will give 


$$
\mathbf{H}_{i}-\mathbf{H}^{\infty}=\frac{1}{3+2 \chi^{\infty}}\left(\mathbf{M}^{\infty}-\mathbf{M}_{i}\right)
$$

where $\mathbf{H}^{\infty}$ and $\mathbf{M}^{\infty}$ denote the macroscopic (average) magnetic field and magnetization and $\chi^{\infty}$ is the macroscopic susceptibility defined as $\chi^{\infty}=$ $M^{\infty} / H^{\infty}$. Applying Equation (13) to the current problem without an applied stress, the field along the magnetization axis in the ferromagnetic phase $\alpha$ becomes

$$
H_{\alpha}=H+\frac{1}{3+2 \chi}\left(M-M_{\alpha}\right)
$$

with $\chi=M / H$. With the magnetic susceptibility of the ferromagnetic phase defined as $\chi_{\alpha}=M_{\alpha} / H_{\alpha}$, it follows that

$$
\frac{H}{H_{\alpha}}=\frac{3+2 \chi+\chi_{\alpha}}{3+3 \chi}
$$

where the volume fraction of the ferromagnetic phase is defined as

$$
f_{\alpha}=\frac{M}{M_{\alpha}}
$$

It should be noted that this definition differs from the classical one given in Equation (3) since true saturation is not achieved. This relationship is most appropriate for low magnetic field levels where the magnetic behavior can be considered as linear. Equation (16) is transformed by introducing the corresponding magnetic fields and susceptibilities

$$
f_{\alpha}=\frac{\chi H}{\chi_{\alpha} H_{\alpha}}
$$

Finally, by combining Equations (15) and (17), $f_{\alpha}$ reads

$$
f_{\alpha}=\frac{\chi\left(3+2 \chi+\chi_{\alpha}\right)}{\chi_{\alpha}(3+3 \chi)}
$$

However, this expression is only valid considering the initial susceptibility (or linear behavior). By recognizing that $\chi=\frac{1}{3} A_{s} \mu_{0} M^{s 2}$ and $\chi_{\alpha}=$ $\frac{1}{3} A_{s} \mu_{0} M_{\alpha}^{s 2}$ following the theoretical development in Section 2.3.1 and considering $\chi \gg 1$, the volume fraction of ferromagnetic phase is expressed as 


$$
f_{\alpha} \approx\left(\frac{M^{s}}{M_{\alpha}^{s}}\right)^{2}
$$

The volume fraction of ferromagnetic phase thus obtained provides a second estimation of $f_{\alpha+\alpha^{\prime}}$ with the hypothesis that the ferromagnetic regions do not interact closely. This description is only partially true and a more appropriate estimation may be defined between expressions (3) and (19). We propose the use of a combination of the two estimations using a ratio parameter $\kappa$, which leads to

$$
f_{\alpha+\alpha^{\prime}}=\frac{M^{s}}{M_{\alpha}^{s}}+\kappa\left[\left(\frac{M^{s}}{M_{\alpha}^{s}}\right)^{2}-\frac{M^{s}}{M_{\alpha}^{s}}\right]
$$

for the case without applied stress and

$$
f_{\alpha+\alpha^{\prime}}=\frac{M_{\sigma}^{s}}{(1+\eta \sigma) M_{\alpha}^{s}}\left[1+\kappa\left(\frac{M^{s}}{(1+\eta \sigma) M_{\alpha}^{s}}-1\right)\right]
$$

to account for the Villari effect and measured saturation $M_{\sigma}^{s}$. The $\kappa$ parameter is identified via a magnetic measurement on a partially transformed material (in the unstrained state) with a known volume fraction of ferromagnetic material and was determined to be $\frac{2}{3}$ in the current case.

If Equation (21) is used instead of the ratio given in Equation (3), this correction improves measurement accuracy when high volume fractions of retained austenite are present.

In the current study, this method is implemented for in-situ tensile testing at a strain rate of $5 \cdot 10^{-4} \mathrm{~s}^{-1}$ on flat tensile samples for each intercritical annealing temperature $T_{I A}$. While the quasi-static strain rate used herein is hardly representative of the strain rates used in forming operations, the intention is to demonstrate the utility of the method in performing high-frequency measurements of the retained austenite volume fraction. A successful implementation of this method would make it possible to perform further studies at much higher strain rates for which no method of measuring the retained austenite fraction during straining exists.

\section{Results and Discussion}

\subsection{Tensile Characterization}

The samples' mechanical behavior in uniaxial tension varied significantly with intercritical annealing temperature. Such a variation of mechanical 
337 behavior with respect to intercritical annealing temperature is frequently 338 observed in Medium Mn steels even for differences in $T_{I A}$ of only $5-10^{\circ} \mathrm{C}$ 339 [4-8]. As seen in Figure $7(\mathrm{a})$, the samples annealed at $740^{\circ} \mathrm{C}$ and $760^{\circ} \mathrm{C}$ 340 showed significant yield point elongation in the form of a Lüders plateau. 341 The samples annealed at $780^{\circ} \mathrm{C}$ did not show a traditional Lüders band, but 342 rather a sort of pseudo-plateau during which stress is not constant. Tests 343 using DIC later confirmed that there is no strain localization in samples 344 annealed at $780^{\circ} \mathrm{C}$, so the pseudo-plateau observed on its tensile curve is not 345 a Lüders effect. 


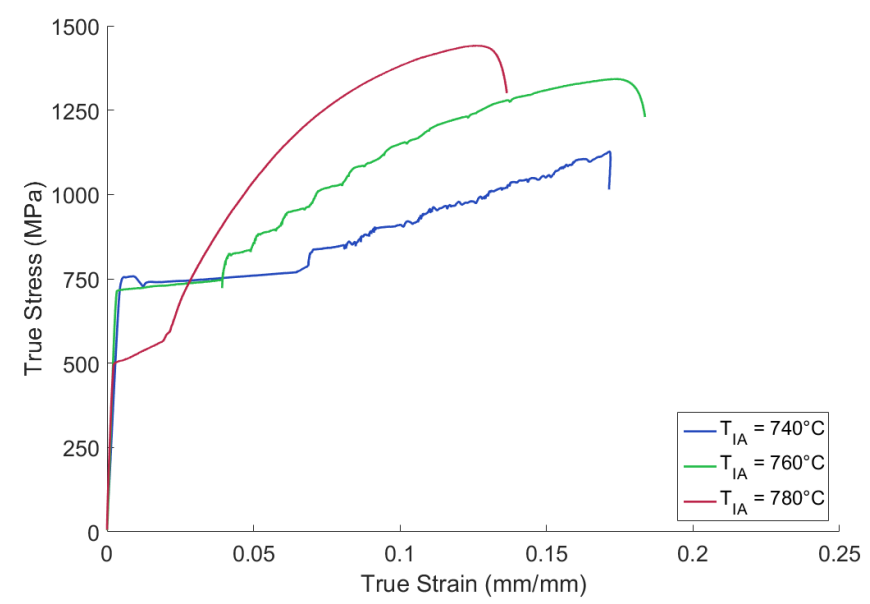

(a)

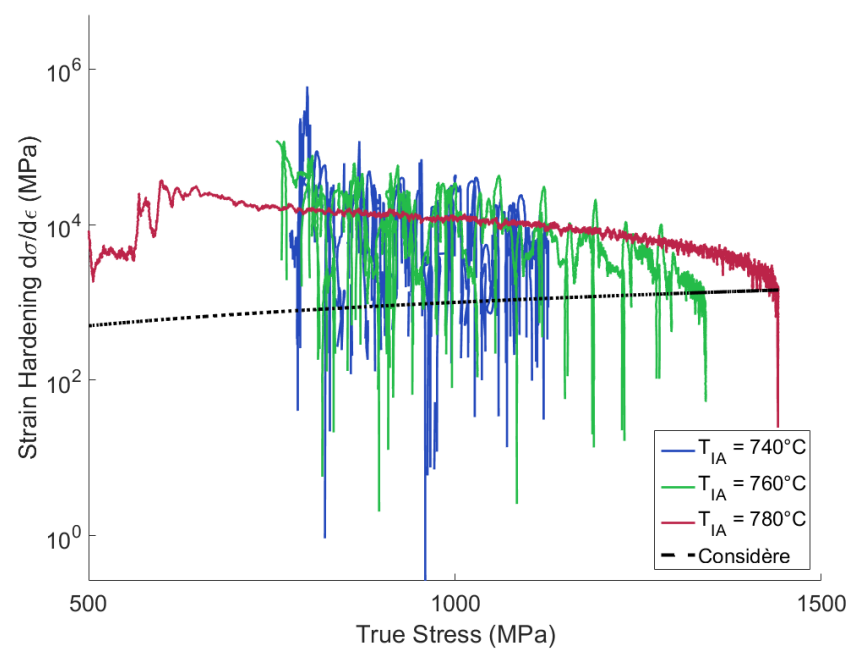

(b)

Figure 7: (a) Representative true stress - true strain curves for each intercritical annealing temperature employed. (b) Strain hardening rate $d \sigma / d \epsilon$ plotted as a function of the true stress $\sigma$ for each intercritical annealing temperature $T_{I A}$. The Considère criterion, wherein the strain hardening rate is equal to the applied true stress, is included as well to show that localizations can bring the hardening rate below this criterion and lead to premature necking.

$346 \quad$ Following yielding, the samples annealed at $740^{\circ} \mathrm{C}$ and $760^{\circ} \mathrm{C}$ showed a 347 form of jerky flow that manifested itself as a series of strain bands once the 
Lüders band had fully propagated across the sample. Due to the difference in the gauge lengths between the sample and the extensometer (i.e. $60 \mathrm{~mm}$ and $12.5 \mathrm{~mm}$, respectively) the jerky flow took the form of "steps" on the tensile curve. Fewer steps were observed for the samples annealed at $740^{\circ} \mathrm{C}$ than $760^{\circ} \mathrm{C}$, but they were generally longer than the steps seen for $760^{\circ} \mathrm{C}$. The sample annealed at $780^{\circ} \mathrm{C}$ did not show any observable jerky flow behavior. The strain hardening of the samples, shown in Figure 7(b) was characterized using the derivative of the tensile curve, $d \sigma / d \epsilon$, plotted against the true stress. Approximating $d \sigma / d \epsilon$ with a trapezoidal method will naturally create a very noisy signal, so a Savitsky-Golay filter [44] was applied to smooth out the data. It can be seen that the samples annealed at $740^{\circ} \mathrm{C}$ and $760^{\circ} \mathrm{C}$ exhibited significant serrations in the strain hardening curves that were not observed in the sample annealed at $780^{\circ} \mathrm{C}$. These serrations are very similar to those previously observed $[5,7,17]$ and are expected to come from the same phenomenon-strain bands due to a sort of dynamic strain aging effectobserved in other Medium Mn alloys. Such serrations are undesirable for forming operations because passing below the Considére criterion can lead to premature necking if the relaxation associated with a given strain band is significant. The jerky flow in samples annealed at $740^{\circ} \mathrm{C}$ and $760^{\circ} \mathrm{C}$ was further characterized using DIC.

\subsection{Digital Image Correlation}

Strain maps obtained by DIC showed two distinct phenomena, namely, a clear Lüders band at yielding, followed by a series of other strained bands that were primarily propagative. It can be seen in Figure 8 that for the sample annealed at $740^{\circ} \mathrm{C}$, several propagative PLC-type bands move across the gauge length of the specimen and always in the opposite direction of the initial Lüders band. The strain increment everywhere outside the band is very small. In the sample annealed at $760^{\circ} \mathrm{C}$, a Lüders band is once again observed, but the subsequent bands are much less continuous. Bands frequently will stop suddenly and another band appears elsewhere along the length of the sample, as seen in Figure 9. 


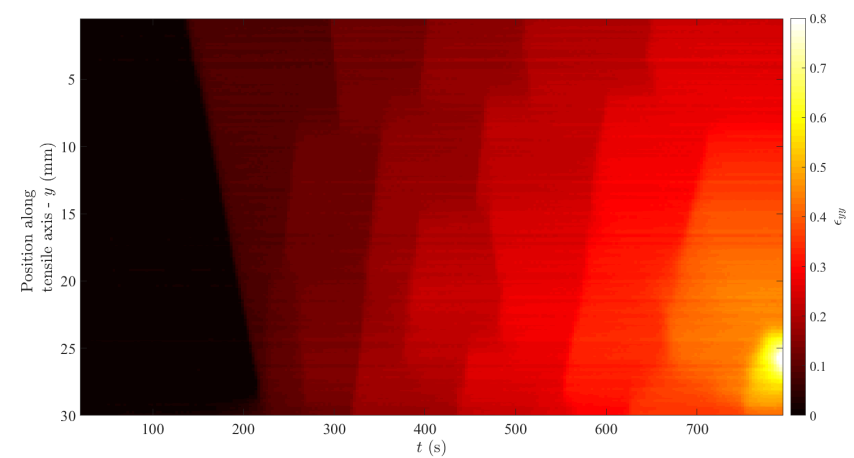

(a)

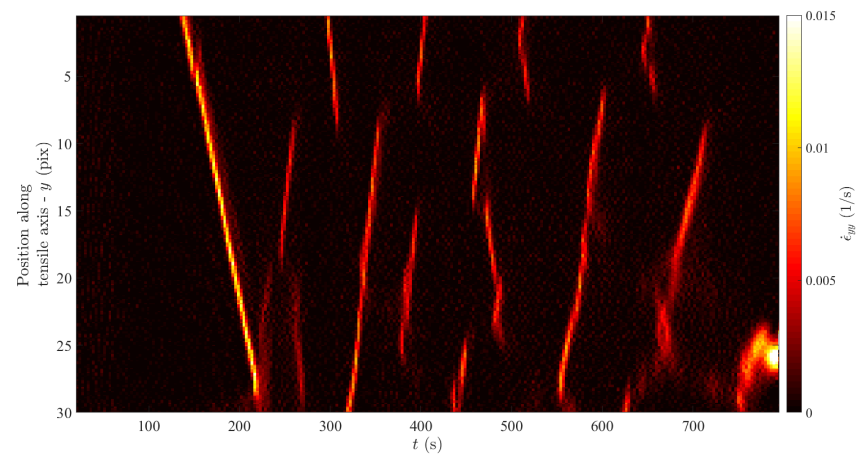

(b)

Figure 8: Spatiotemporal DIC measurements of (a) nominal longitudinal strain and (b) longitudinal strain rate measurements for annealing at $740^{\circ} \mathrm{C}$. The horizontal axis is the time in seconds, the vertical axis represents the position along the tensile direction, and the color bar represents either the total strain in the tensile direction or the strain rate $(1 / \mathrm{s})$ in the tensile direction. 


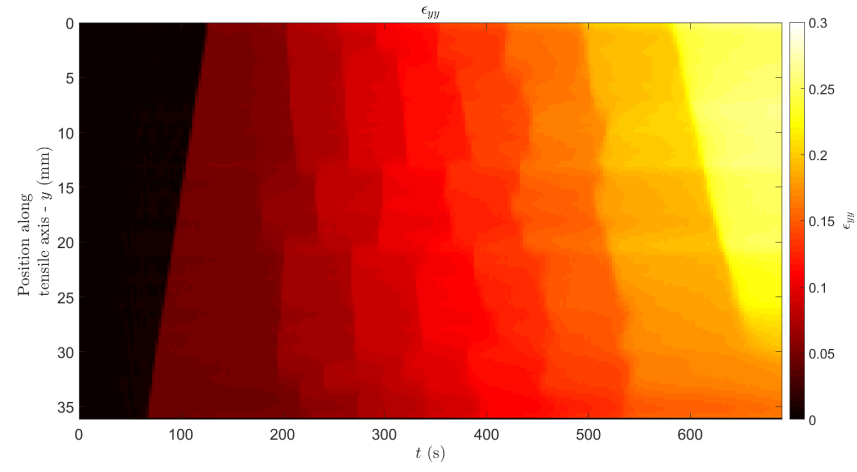

(a)

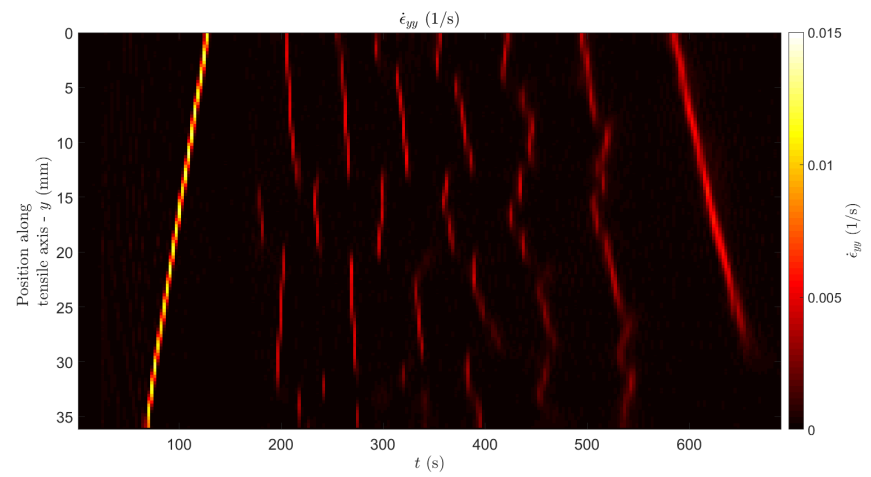

(b)

Figure 9: Spatiotemporal DIC measurements of (a) nominal longitudinal strain and (b) longitudinal strain rate measurements for annealing at $760^{\circ} \mathrm{C}$. The horizontal axis is the time in seconds, the vertical axis represents the position along the tensile direction, and the color bar represents either the total strain in the tensile direction or the strain rate $(1 / \mathrm{s})$ in the tensile direction.

The bands themselves were characterized by using ImageJ to analyze images of the strain increment field for the component along the loading direction $\Delta \varepsilon_{y y}$ and plotting several line profiles along the loading direction. Using the peaks in the line profiles, the band width and inclination angle from the loading direction were calculated for both the Lüders bands and the subsequent PLC-type bands. The PLC-type bands that appeared in the sample annealed at $760^{\circ} \mathrm{C}$ were too random to obtain reliable measurements of their propagation speed and width, so values are only presented for the sample annealed at $740^{\circ} \mathrm{C}$. Table 1 summarizes the results of the band characterization (note that the velocities given for PLC bands is the average of 


\begin{tabular}{rrccc}
\hline & & Velocity $(\mathbf{m m} / \mathbf{s})$ & Band Width $(\mathbf{m m})$ & Inclination $\theta\left(^{\circ}\right)$ \\
\hline $740^{\circ} \mathrm{C}$ & Lüders & $0.381 \pm 0.008$ & $0.605 \pm 0.033$ & $66.4 \pm 0.496$ \\
& PLC & $0.583 \pm 0.084$ & $0.770 \pm 0.042$ & $69.8 \pm 0.605$ \\
$760^{\circ} \mathrm{C}$ & Lüders & $0.623 \pm 0.003$ & $0.246 \pm 0.044$ & $69.3 \pm 0.800$ \\
\hline
\end{tabular}

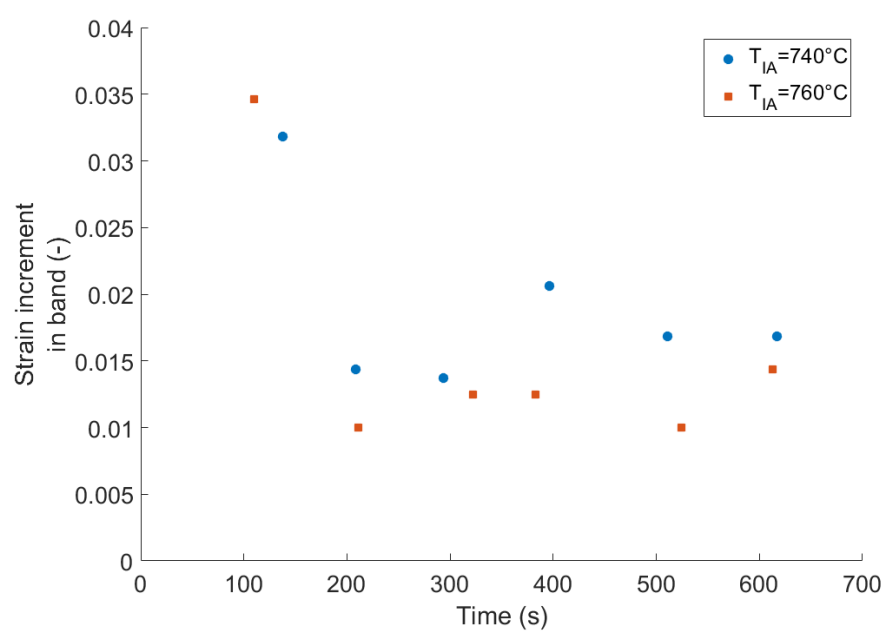

Figure 10: Plot of the longitudinal strain increment in each band as a function of time for samples annealed at $740^{\circ} \mathrm{C}$ and $760^{\circ} \mathrm{C}$. The stark difference in magnitude between the first band and those that follow suggests two different underlying mechanisms. It is proposed that there is an initial Lüders band followed by PLC-type bands. band for both intercritical annealing temperatures. The strain in the first band is significantly higher than in subsequent band, suggesting that two different phenomena are responsible for the first band and those that follow.

Table 1: DIC strain band characterization

As can be seen in Figure 11, the velocity of the PLC-type bands decreased roughly linearly with total strain while the strain rate in the band remained constant. Both this trend and the observed velocities are in agreement with previous experiments [29]. Comparing these results to those published for aluminum alloys or TWIP steels exhibiting the PLC effect, it can be seen that for propagative type-A PLC bands the band velocity also linearly decreases with strain at a rate roughly one order of magnitude slower than observed here [45-47]. It is thus proposed that the bands observed in Medium

each PLC band for that $\left.T_{I A}\right)$. Figure 10 shows the strain magnitude in each 
Mn steels are likely type-A PLC bands. The difference in rate of band velocity decrease with strain could be due to chemical composition (via the solute concentrations and concentration gradients), and interaction with the martensitic transformation, or both. PLC resulting from an interaction between dislocations and Mn-C clusters has been previously proposed by Jung and DeCooman [48], who suggested that the movement of C atoms in C-Mn clusters between octahedral and tetrahedral interstices could lock partial dislocations in stacking faults. Hickel et al. [49] also confirmed a strong effect of C-Mn clusters on SFE and showed that the active deformation mechanism can locally redistribute solutes and thus significantly change the local SFE.

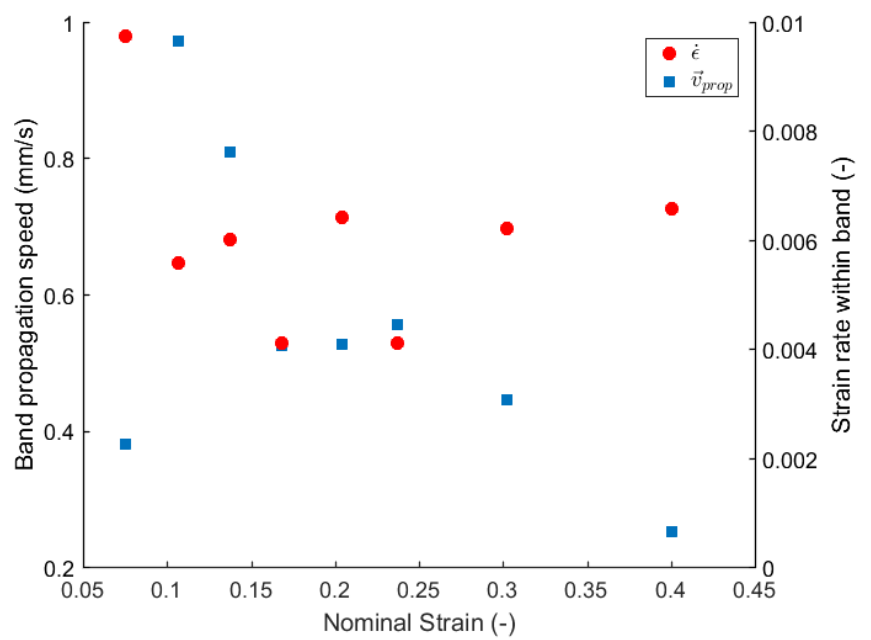

Figure 11: Calculated band propagation velocities and the strain rate within each band plotted as a function of the nominal strain for the sample annealed at $740^{\circ} \mathrm{C}$. The velocities of the PLC-type bands decrease roughly linearly with strain while the strain rate in the band remains more or less constant for bands occurring after the Lüders band.

\subsection{Magnetic Measurements}

The in-situ measurement of sample magnetization throughout tensile testing allowed the retained austenite volume fraction (via the combined volume fractions of ferrite and martensite) to be calculated. This method provided continuous phase volume fraction measurements over the $37 \mathrm{~mm}$ of the sample's gauge length that is contained within the driving coil, thereby ensuring a very large measurement volume that is statistically representative of the steel's microstructure. Additionally, because of the difference 
in the magnetic measurement length and the tensile length of the sample $(60 \mathrm{~mm})$, if any strain localizations are present that induce TRIP their effect should produce plateaus in the measurement of the austenite volume fraction as a function of strain. Figure 12 presents the true stress and retained austenite volume fraction as a function of true plastic strain as calculated from the crosshead displacement for each intercritical annealing temperature. The plastic strain is calculated by assuming that the tensile machine deforms purely elastically, making it possible to simply remove the elastic strain $\epsilon^{e}=\sigma / E$. 


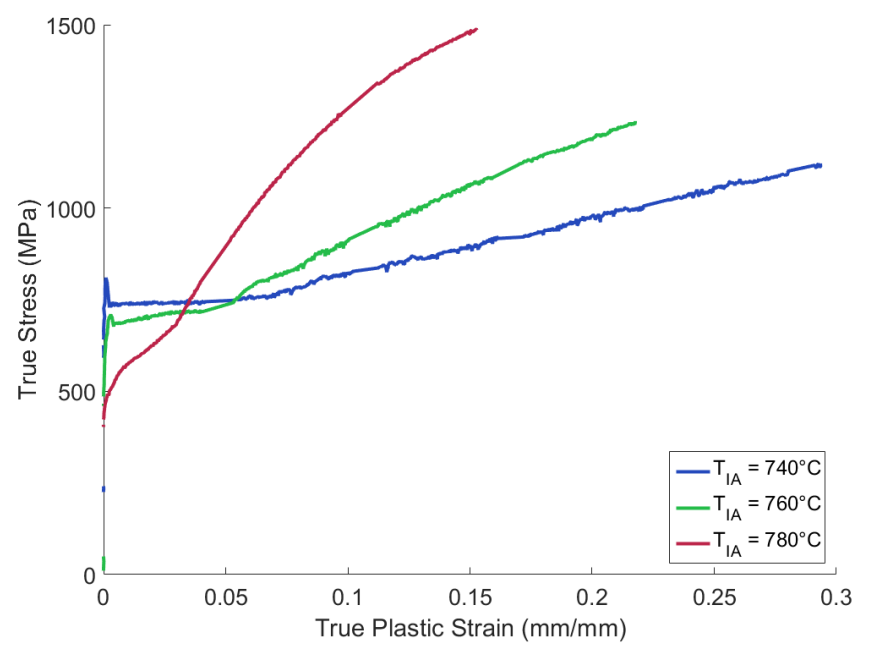

(a)

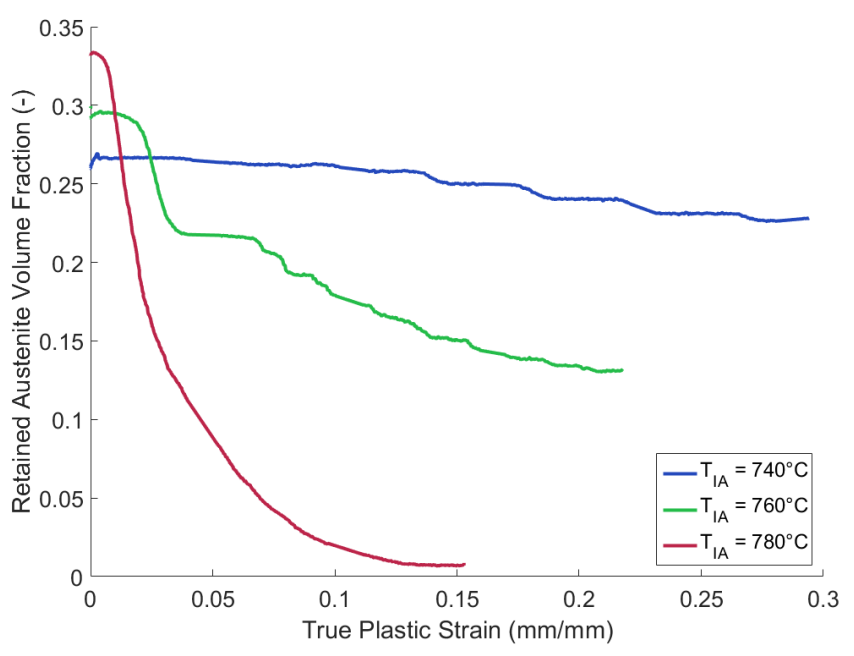

(b)

Figure 12: Plots of (a) true stress and (b) retained austenite volume fraction vs. true plastic strain for each intercritical annealing temperature. The same steps previously observed in tensile data are again seen in the retained austenite volume fraction, suggesting that the TRIP effect coincides with the passage of strain bands. Both plots share identical strain axes.

An important clarification must be made. Because the magnetic measurement system completely encloses the tensile sample, the strain in the sample 
was not directly measured during tensile testing. Instead, the true plastic strain is used in order to eliminate the elastic strain in the tensile machine as recorded by the crosshead displacement. The curves presented for each intercritical annealing temperature in Figure 12 are thus from two different samples and, while the dispersion in tensile data was small, the indications of banding will not be identical in two curves for the same annealing temperature. This effect aside, it is clear that in the magnetic data the retained austenite volume fraction decreases sharply at several points, creating the same "steps" that were previously seen in tensile data using an extensometer. This strongly suggests that the passage of strain bands and the TRIP effect are, in fact, coincident.

Previous work by Wang et al. [29] showed that the TRIP effect occurred during the Lüders plateau, but that little or no transformation occurred during PLC. It has also been shown by Sun et al. [28] that some transformation does occur during PLC. The current work then confirms Sun et al.'s result that TRIP occurs both during Lüders deformation and PLC. It is possible that when the amount of austenite transformed by a single band is low (i.e. $\approx 1-2 \%$ ), the heat given off by the reaction is "drowned out" by the adiabatic heating (even at quasi-static strain rates) in the band and thus difficult to distinguish by infrared imagery. It is also possible that the retained austenite in Wang et al.'s steel was simply stable enough to not transform noticeably. This point is important to address because the origin of the PLC-type localizations is, as mentioned in Section 3.2, a point of contention currently and if one is able to have PLC without a phase transformation in these steels it would indicate that the PLC effect is not initiated by the phase transformation.

The correction of the stress effect on magnetization allowed stress-induced and strain-induced martensite transformations to be separated and showed that no stress-induced transformation occurred during elastic deformation. Figure 13 shows the sample magnetization vs. true stress for each intercritical annealing temperature. In all samples the magnetization remains constant in the elastic domain, proving that no stress-induced transformation occurs during elasticity and that the first martensitic transformation occurs only after the onset of plasticity. 


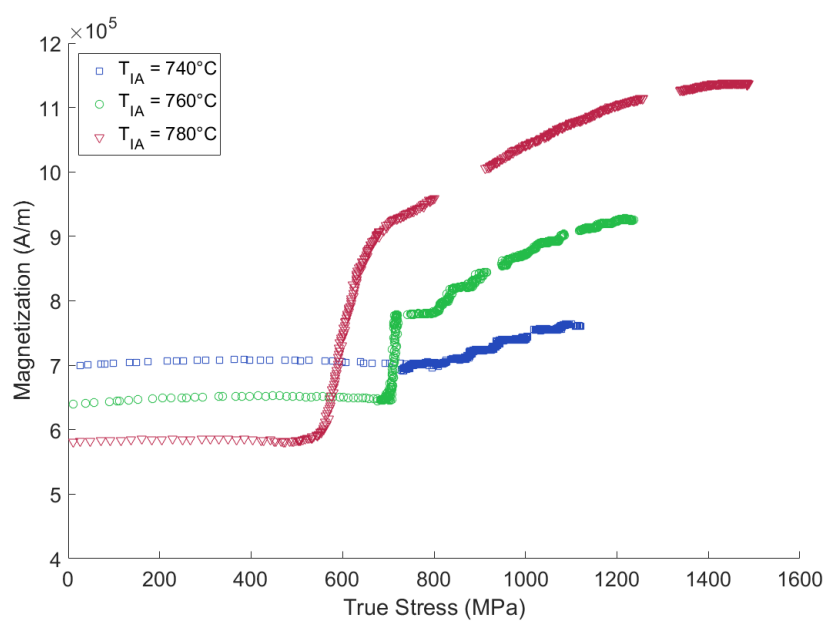

Figure 13: Sample magnetization (with stress correction) plotted as a function of true stress shows that during elasticity, the magnetization remained constant meaning that there is no stress-induced transformation before yielding.

The changes of the austenite volume fraction $\left(f_{\gamma}=1-f_{\alpha+\alpha^{\prime}}\right)$ as calculated from magnetization measurements were used to fit the parameters of an Olson-Cohen model [50]

$$
f_{\alpha+\alpha^{\prime}}=1-\exp \left(-\beta\left(1-\exp \left(-\alpha \epsilon^{p}\right)^{n}\right)\right.
$$

where the $\alpha$ parameter is related to both the number of shear bands and the stacking fault energy of the austenite, $\beta$ accounts for the probability of a martensite nucleus to form, which is in turn defined by the driving force for the phase transformation. The exponent $n$ is usually considered a material constant, but is not fixed in the current case due to the varying composition of the austenite for each $T_{I A}$. Perhaps surprisingly, the model can be fit to the measured austenite volume fraction change fairly well even when strain localizations are present as can be seen in Figure 14. This provides a set of effective Olson-Cohen parameters, given in Table 2, that are valid in a macroscopic sense for a given microstructure and austenite stability. Estimations of the martensite start temperature, $M_{S}$, are also provided in Table 2 . 


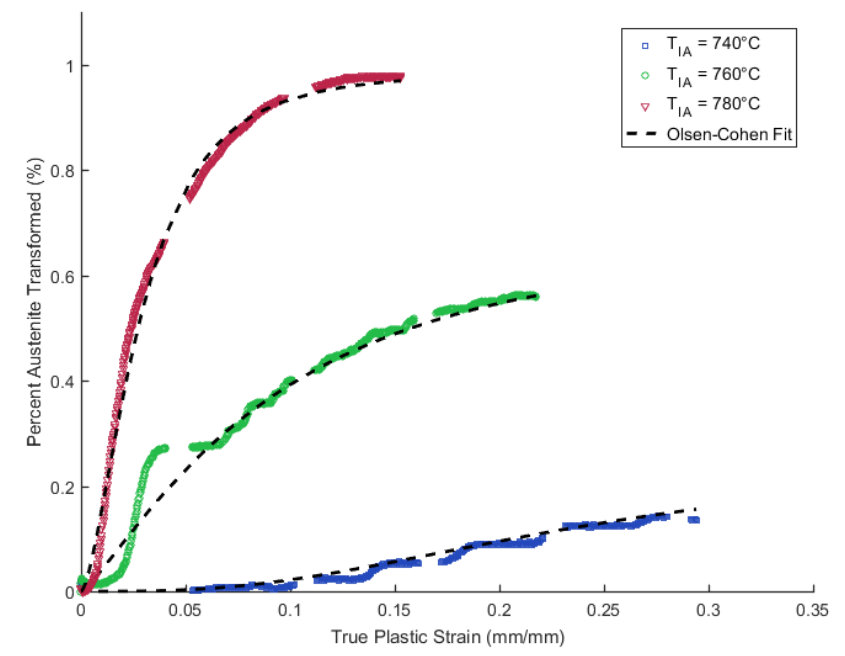

Figure 14: Experimental transformation rates of retained austenite in alloys annealed at $740^{\circ} \mathrm{C}, 760^{\circ} \mathrm{C}, 780^{\circ} \mathrm{C}$ (symbols), fit with a standard Olsen-Cohen model (dashed lines).

Table 2: Macroscopic Olson-Cohen Parameters

\begin{tabular}{ccccc}
\hline$T_{I A}\left({ }^{\circ} C\right)$ & $\alpha$ & $\beta$ & $n$ & $M_{S}\left({ }^{\circ} C\right)$ \\
\hline 740 & 7.52 & 0.27 & 3.90 & -7.5 \\
760 & 8.05 & 1.04 & 1.25 & 26 \\
780 & 14.0 & 4.25 & 1.58 & 58 \\
\hline
\end{tabular}

Examining the trends in the Olson-Cohen model's fitting parameters, one could begin to explain the differences in TRIP kinetics between samples for different intercritical annealing temperatures. Considering the $\alpha$ and $\beta$ parameters, Olson and Cohen [50] suggest that they should increase as the test temperature decreases for a given material. Thus, $\alpha$ and $\beta$ should increase as austenite stability decreases, which is the trend observed herein. However $n$ should have a maximum at an intermediate test temperature, but instead there is a minimum at $760^{\circ} \mathrm{C}$ and a large discrepancy at $740^{\circ} \mathrm{C}$ compared to the other annealing temperatures. The exponent $n$ is related to the composition of the material, which should not vary to such a degree given that only the intercritical annealing temperature changes and not the nominal composition. As a result, it seems that the base Olson-Cohen model is sufficient to confirm trends in SFE between alloys, but not to truly understand 
differences in TRIP kinetics between two different austenite compositions. Alternatively, it is also possible that the trends in the model parameters are not the same when varying test temperature (as Olson and Cohen did) or austenite composition/volume fraction (as in the current study). In any case, one could further investigate the interactions between phases using numerical models parameterized using the kinetics obtained for each intercritical annealing temperature in these experiments. Doing so would allow for a better description of the true kinetics since microstructure-level strains and stresses would be available.

\section{Conclusions}

A system for measuring the retained austenite volume fraction via sample magnetization has been developed and used in-situ during tensile testing. This method provides very rapid measurements of the retained austenite fraction (here $2 \mathrm{~Hz}$ ) relative to other methods such as XRD or EBSD and enables measurements to be conducted without pausing or otherwise interrupting the tensile test. However, the measurement system encloses the sample, so the strain must either be calculated from the crosshead displacement or measured with a strain gauge (extensometers or DIC methods cannot be used on a sample upon which these magnetic measurements are made).

This system was employed to study the effects of intercritical annealing temperature and strain instabilities on the TRIP effect present in samples of a Fe-0.2C-5Mn-2.5Al Medium Mn steel that had been intercritically annealed at $740^{\circ} \mathrm{C}, 760^{\circ} \mathrm{C}$, and $780^{\circ} \mathrm{C}$. There was a clear difference in the rate of martensitic transformation for different intercritical annealing temperatures, though it is not understood exactly how the micromechanics of the TRIP effect are altered by varying $T_{I A}$. Plateaus corresponding to the passage of strain bands appear both in tensile data measured with an extensometer and in magnetization data. This indicates that the martensitic transformation and the passage of strain bands occur simultaneously.

DIC analyses of the strain bands present in samples annealed at $740^{\circ} \mathrm{C}$ and $760^{\circ} \mathrm{C}$ showed that the Lüders and post-Lüders bands are from two separate phenomena and that, because the band velocity in the sample annealed at $740^{\circ} \mathrm{C}$ decreased linearly with strain at a rate similar to that observed in Al and TWIP steels exhibiting PLC [23-25], the post-Lüders bands are likely to be propagative type-A PLC bands. The micromechanical origin of 
the PLC effect is unknown and has been hypothesized to be related to either C-Mn clusters or the martensite transformation itself $[5,7,17]$.

The experimental procedures established were intended to study the effects of $T_{I A}$ and strain instabilities on TRIP, but provide a tool that can be extended to use in the optimization of Medium Mn TRIP steels in general. The magnetic measurement system is capable of measuring the retained austenite fraction in-situ even for the high strain rates used in forming operations or for applied loads other than unidirectional tension. The data obtained could be used to calibrate a micromechanical model of TRIP kinetics and thus enable the metallurgist to predict the martensite transformation rates for a given microstructure and strain rate. The extension of this method to such a purpose is underway as well as studies of the effect of the applied strain rate on transformation kinetics.

\section{Acknowledgements}

The authors would like to thank Dr. Denis Solas from Université Paris Sud for his expertise with XRD measurements. This study has been performed within the framework of the ANR 13-RMNP-0002 MedMnAl Steels funded by the Agence Nationale de la Recherche (ANR). This project is supported by the competitive cluster "Materalia".

\section{References}

[1] T. Pardoen, F. Delannay, On the Role of Martensitic Transformation on Damage and Cracking Resistance in TRIP-Assisted Multiphase Steels, Acta Materialia 49 (2001) 139-152.

[2] J. Chiang, B. Lawrence, J. D. Boyd, A. K. Pilkey, Effect of microstructure on retained austenite stability and work hardening of TRIP steels, Materials Science and Engineering A 528 (2011) 4516-4521.

[3] J. Park, M. Kang, S. S. Sohn, J.-S. Kim, H. S. Kim, W. T. Cho, S. Lee, Tensile properties of cold-rolled TWIP-cored three-layer steel sheets, Materials Science and Engineering: A 686 (2017) 160-167.

[4] A. Arlazarov, M. Gouné, O. Bouaziz, A. Hazotte, G. Petitgand, P. Barges, Evolution of microstructure and mechanical properties of medium Mn steels during double annealing, Materials Science and Engineering A 542 (2012) 31-39. 
[5] Z. Cai, H. Ding, R. Misra, Z. Ying, Austenite stability and deformation behavior in a cold-rolled transformation-induced plasticity steel with medium manganese content, Acta Materialia 84 (2015) 229-236.

[6] J. Chen, M. Lv, S. Tang, Z. Liu, G. Wang, Correlation between mechanical properties and retained austenite characteristics in a low-carbon medium manganese alloyed steel plate, Materials Characterization 106 (2015) 108-111.

[7] P. J. Gibbs, E. De Moor, M. J. Merwin, B. Clausen, J. G. Speer, D. K. Matlock, Austenite stability effects on tensile behavior of manganeseenriched-austenite transformation-induced plasticity steel, Metallurgical and Materials Transactions A: Physical Metallurgy and Materials Science 42 (2011) 3691-3702.

[8] S. Lee, B. C. De Cooman, Tensile Behavior of Intercritically Annealed Ultra-Fine Grained 8\% Mn Multi-Phase Steel, Steel Research International 86 (2015) 1170-1178.

[9] Z. Tang, R. Misra, M. Ma, N. Zan, Z. Wu, H. Ding, Deformation twinning and martensitic transformation and dynamic mechanical properties in Fe0.07C23Mn3.1Si2.8Al TRIP/TWIP steel, Materials Science and Engineering: A 624 (2015) 186-192.

[10] K. Rahman, V. Vorontsov, D. Dye, The effect of grain size on the twin initiation stress in a TWIP steel, Acta Materialia 89 (2015) 247-257.

[11] L. Zhao, N. van Dijk, E. Bruck, J. Sietsma, S. van der Zwaag, Magnetic and X-ray diffraction measurements for the determination of retained austenite in TRIP steels, Materials Science \& Engineering A 313 (2001) $145-152$.

[12] P. J. Jacques, S. Allain, O. Bouaziz, A. De, A.-F. Gourgues, B. M. Hance, Y. Houbaert, J. Huang, A. Iza-Mendia, S. E. Kruger, M. Radu, L. Samek, J. Speer, L. Zhao, S. van der Zwaag, On measurement of retained austenite in multiphase TRIP steels results of blind round robin test involving six different techniques, Materials Science and Technology 25 (2009) 567-574. 
[13] M. Moallemi, A. Kermanpur, A. Najafizadeh, A. Rezaee, H. S. Baghbadorani, P. D. Nezhadfar, Deformation-induced martensitic transformation in a 201 austenitic steel: The synergy of stacking fault energy and chemical driving force, Materials Science and Engineering A 653 (2016) 147-152.

[14] A. M. Beese, D. Mohr, Identification of the Direction-Dependency of the Martensitic Transformation in Stainless Steel Using In Situ Magnetic Permeability Measurements, Experimental Mechanics 51 (2011) 667676.

[15] M. Radu, J. Valy, A. Gourgues, F. Le Strat, A. Pineau, Continuous magnetic method for quantitative monitoring of martensitic transformation in steels containing metastable austenite, Scripta Materialia 52 (2005) 525-530.

[16] P. J. Gibbs, B. C. De Cooman, D. W. Brown, B. Clausen, J. G. Schroth, M. J. Merwin, D. K. Matlock, Strain partitioning in ultra-fine grained medium-manganese transformation induced plasticity steel, Materials Science and Engineering A 609 (2014) 323-333.

[17] S. Lee, B. C. De Cooman, Effect of the Intercritical Annealing Temperature on the Mechanical Properties of 10Pct Mn Multi-phase Steel, Metallurgical and Materials Transactions A 45 (2014) 5009-5016.

[18] G. Besnard, F. Hild, S. Roux, "Finite-Element" Displacement Fields Analysis from Digital Images: Application to Portevin-Le Chatelier Bands, Experimental Mechanics 46 (2006) 789-803.

[19] J. Kang, D. Wilkinson, M. Jain, J. Embury, A. Beaudoin, S. Kim, R. Mishira, A. Sachdev, On the sequence of inhomogeneous deformation processes occurring during tensile deformation of strip cast AA5754, Acta Materialia 54 (2006) 209-218.

[20] H. Halim, D. Wilkinson, M. Niewczas, The Portevin-Le Chatelier (PLC) effect and shear band formation in an AA5754 alloy, Acta Materialia 55 (2007) 4151-4160.

[21] A. Benallal, T. Berstad, T. B. rvik, O. Hopperstad, I. Koutiri, R. N. de Codes, An experimental and numerical investigation of the behaviour 
of AA5083 aluminium alloy in presence of the Portevin-Le Chatelier effect, International Journal of Plasticity 24 (2008) 1916-1945.

[22] J. Réthoré, G. Besnard, G. Vivier, F. Hild, S. Roux, Experimental investigation of localized phenomena using digital image correlation, Philosophical Magazine 88 (2008) 3339-3355.

[23] J. Zdunek, T. Brynk, J. Mizera, Z. Pakiela, K. Kurzydlowski, Digital Image Correlation investigation of Portevin-Le Chatelier effect in an aluminium alloy, Materials Characterization 59 (2008) 1429-1433.

[24] P. Zavattieri, V. Savic, L.G. Hector Jr., J.R. Fekere, W. Tong, Y. Xuan, Spatio-temporal characteristics of the Portevin-Le Chatelier effect in austenitic steel with twinning induced plasticity, International Journal of Plasticity 25 (2009) 2298-2330.

[25] J. Coër, P. Manach, H. Laurent, M. Oliveira, L. Menezes, PiobertLüders plateau and Portevin-Le Chatelier effect in an Al-Mg alloy in simple shear, Mechanics Research Communications 48 (2013) 1-7.

[26] J. Min, J. Lin, B. Sun, Effect of strain rate on spatio-temporal behavior of portevin-le chatelier bands in a twinning induced plasticity steel, Mechanics of Materials 68 (2014) 164-175.

[27] T. Cheng, X. Xu, Y. Cai, S. Fu, Y. Gao, Y. Su, Y. Zhang, Q. Zhang, Investigation of Portevin-Le Chatelier effect in 5456 Al-based alloy using digital image correlation, Optics and Lasers in Engineering 65 (2015) 89-92.

[28] B. Sun, N. Vanderesse, F. Fazeli, C. Scott, J. Chen, P. Bocher, M. Jahazi, S. Yue, Discontinuous strain-induced martensite transformation related to the Portevin-Le Chatelier effect in a medium manganese steel, Scripta Materialia 133 (2017) 9-13.

[29] X. G. Wang, L. Wang, M. X. Huang, Kinematic and thermal characteristics of Lüders and Portevin-Le Châtelier bands in a medium Mn transformation-induced plasticity steel, Acta Materialia 124 (2017) 1729. 
[30] M. Bach, N. Broll, A. Cornet, L. Gaide, Diffraction X en traitements thermiques: dosage de l'austénite résiduelle par diffraction des rayons $\mathrm{X}$, journal $=$ Journal de Physique IV Colloque 06 (1996) 887-895.

[31] Z. Tomicevc, F. Hild, S. Roux, Mechanics-aided digital image correlation, The Journal of Strain Analysis for Engineering Design 48 (2013) $330-343$.

[32] M. Abramoff, P. Magalhaes, S. Ram, Image processing with ImageJ, Biophotonics International 11 (2004) 36-42.

[33] C. Bormio-Nunes, O. Hubert, Piezomagnetic behavior of FeAlB alloys, Journal of Magnetism and Magnetic Materials 393 (2015) 404-418.

[34] O. Hubert, L. Daniel, Energetical and multiscale approaches for the definition of an equivalent stress for magneto-elastic couplings, Journal of Magnetism and Magnetic Materials 323 (2011) 1766-1781.

[35] A. Vinogradov, A. Lazarev, M. Linderov, A. Weidner, H. Biermann, Kinetics of deformation processes in high-alloyed cast transformationinduced plasticity/twinning-induced plasticity steels determined by acoustic emission and scanning electron microscopy: Influence of austenite stability on deformation mechanisms, Acta Materialia 61 (2013) 2434-2449.

[36] S. S. Hecker, M. G. Stout, K. P. Staudhammer, J. L. Smith, Effects of Strain State and Strain Rate on Deformation-Induced Transformation in 304 Stainless Steel: Part I. Magnetic Measurements and Mechanical Behavior, Metallurgical Transactions A 13 (1982) 619-626.

[37] O. Perevertov, Influence of the applied elastic tensile and compressive stress on the hysteresis curves of Fe-3\% Si non-oriented steel, Journal of Magnetism and Magnetic Materials 428 (2017) 223-228.

[38] O. Hubert, S. Lazreg, Two phase modeling of the influence of plastic strain on the magnetic and magnetostrictive behaviors of ferromagnetic materials, Journal of Magnetism and Magnetic Materials 424 (2017) 421-442.

[39] D. Vanoost, S. Steentjes, J. Peuteman, G. Gielen, H. D. Gersem, D. Pissoort, Magnetic hysteresis at the domain scale of a multi-scale material 
model for magneto-elastic behaviour, Journal of Magnetism and Magnetic Materials 414 (2016) 168-179.

[40] S. Lazreg, O. Hubert, A multidomain modeling of the magnetoelastic behavior for nondestructive evaluation, Journal of Applied Physics 109 (2011) 07E508.

[41] X. J. Hao, W. Yin, M. Strangwood, A. J. Peyton, P. F. Morris, C. L. Davis, Modelling the electromagnetic response of two-phase steel microstructures, NDT and E International 43 (2010) 305-315.

[42] J. Eshelby, The determination of the elastic field of an ellipsoidal inclusion, and related problems, Proceedings of the Royal Society (1957).

[43] F. Mballa-Mballa, O. Hubert, S. Lazreg, P. Meilland, Multidomain modelling of the magneto-mechanical behaviour of dual-phase steels, in: 18th World Conference on Nondestructive Testing, April, pp. 16-20.

[44] A. Savitsky, M. Golay, Smoothing and Differentiation of Data by Simplified Least Squares Procedures, Journal of Analytical Chemistry 36 (1964) 1627-1639.

[45] K. Renard, S. Ryelandt, P. J. Jacques, Characterisation of the PortevinLe Châtelier effect affecting an austenitic TWIP steel based on digital image correlation, Materials Science and Engineering A 527 (2010) 29692977.

[46] H. Ait-Amokhtar, P. Vacher, S. Boudrahem, Kinematics fields and spatial activity of Portevin-Le Chatelier bands using the digital image correlation method, Acta Materialia 54 (2006) 4365-4371.

[47] H. Ait-Amokhtar, C. Fressengeas, Crossover from continuous to discontinuous propagation in the Portevin-Le Chatelier effect, Acta Materialia 58 (2010) 1342-1349.

[48] I. C. Jung, B. C. De Cooman, Temperature dependence of the flow stress of Fe-18Mn-0.6C-xAl twinning-induced plasticity steel, Acta Materialia 61 (2013) 6724-6735.

[49] T. Hickel, S. Sandlöbes, R. K. W. Marceau, A. Dick, I. Bleskov, J. Neugebauer, D. Raabe, Impact of nanodiffusion on the stacking fault energy in high-strength steels, Acta Materialia 75 (2014) 147-155. 
${ }_{714}$ [50] G. Olson, M. Cohen, Kinetics of Strain-Induced Martensitic Nucleation, 715 Metallurgical Transactions A 6 (1975) 791-795. 